\title{
ON LIMIT SETS FOR THE DISCRETE SPECTRUM OF COMPLEX JACOBI MATRICES
}

\author{
IRYNA EGOROVA AND LEONID GOLINSKII
}

\begin{abstract}
The discrete spectrum of complex Jacobi matrices that are compact perturbations of the discrete laplacian is under consideration. The rate of stabilization for the matrix entries sharp in the sense of order which provides finiteness of the discrete spectrum is found. The Jacobi matrix with the discrete spectrum having the only limit point is constructed. The results can be viewed as the discrete analogs of the well known theorems by B.S. Pavlov about Schrödinger operators on the half line with a complex potential.

Bibliography : 26 references.
\end{abstract}

\section{InTRODUCTION.}

In early 1960th B.S. Pavlov studied the discrete spectrum of Schrödinger operators on the half line

$$
l_{h} y=-y^{\prime \prime}+q(x) y, \quad y^{\prime}(0)-h y(0)=0,
$$

with a complex valued potential $q$ and a complex parameter $h$, and came to the following results $[1,2]$.

Theorem P1. The number of eigenvalues of the operator $l_{h}$ is finite as long as the infinitely differentiable potential $q(x)$ satisfies

$$
|q(x)| \leq C \exp \left(-\varepsilon x^{\frac{1}{2}}\right), \quad x>0
$$

for some $\varepsilon>0$.

Theorem P2. Whatever the numbers $0<\beta<\frac{1}{2}$ and $\lambda>0$ are given, there is a real infinitely differentiable potential $q(x)$ subject for some $\delta>0$ to the bound

$$
|q(x)| \leq C \exp \left(-\delta x^{\beta}\right), \quad x>0
$$

and a complex number $h$ such that operator (1.1) has infinitely many eigenvalues with the only accumulation point $\lambda$.

The main objective of the present paper is to establish the discrete analogs of Pavlov's theorems.

Let

$$
J=\left(\begin{array}{ccccc}
b_{0} & c_{0} & & & \\
a_{0} & b_{1} & c_{1} & & \\
& a_{1} & b_{2} & c_{2} & \\
& & \ddots & \ddots & \ddots
\end{array}\right)
$$

This work is supported in part by INTAS Research Network NeCCA 03-51-6637. 
be a semi-infinite Jacobi matrix with complex entries and let

$$
\lim _{n \rightarrow \infty} a_{n}=\lim _{n \rightarrow \infty} c_{n}=\frac{1}{2}, \quad \lim _{n \rightarrow \infty} b_{n}=0 .
$$

The operator $J$ in the Hilbert space $\ell^{2}\left(\mathbb{Z}_{+}\right), \mathbb{Z}_{+}=\{0,1,2, \ldots\}$, generated by such matrix is thereby a complex perturbation of the discrete laplacian

$$
J_{0}: \quad a_{n}=c_{n}=\frac{1}{2}, \quad b_{n}=0 .
$$

According to the well known Weyl's theorem the spectrum $\sigma(J)$ of $J$ is $\sigma(J)=$ $[-1,1] \bigcup \sigma_{d}(J)$, where a denumerable set $\sigma_{d}(J)$ of eigenvalues of finite algebraic multiplicity, called in what follows the discrete spectrum of the operator $J$, may have accumulation points only on the interval $[-1,1]$. The main object under consideration is a limit set for the discrete spectrum $E_{J}:=\left\{\sigma_{d}(J)\right\}^{\prime}$ for the operators (1.2)-(1.3) .

The following question arises naturally: how to describe the class $\left\{E_{J}\right\}_{J}$ of closed point sets on $[-1,1]$, which can emerge as limit sets for discrete spectra of Jacobi matrices (1.2)-(1.3) or for certain subclasses of such matrices. Our results can be viewed as the first step along the way of solving this general problem. They concern primarily the case of empty limit sets (finite discrete spectrum).

Let us say that $J$ (1.2) belongs to the class $\mathcal{P}(\beta)$ if

$$
\left|a_{n}-\frac{1}{2}\right|+\left|c_{n}-\frac{1}{2}\right|+\left|b_{n}\right| \leq C_{1} \exp \left(-C_{2} n^{\beta}\right), \quad 0<\beta<1, \quad C_{1}, C_{2}>0 .
$$

To formulate our first result recall that the convergence exponent or Taylor-Besicovitch index of a closed point set $F \subset[-1,1]$ is the value

$$
\tau(F):=\inf \left\{\varepsilon>0: \sum_{j=1}^{\infty}\left|l_{j}\right|^{\varepsilon}<\infty\right\}
$$

where $\left\{l_{j}\right\}$ are the adjacent intervals of $F$.

Theorem 1. Let $J \in \mathcal{P}(\beta)$ where $0<\beta<\frac{1}{2}$. Then $E_{J}$ is a closed point set of the Lebesgue measure zero and its convergence exponent satisfies

$$
\operatorname{dim} E_{J} \leq \tau\left(E_{J}\right) \leq \frac{1-2 \beta}{1-\beta},
$$

where $\operatorname{dim} E_{J}$ is the Hausdorff dimension of $E_{J}$. Moreover, if $J \in \mathcal{P}\left(\frac{1}{2}\right)$ then $E_{J}=\emptyset$ i.e. the discrete spectrum is finite.

The statement about finiteness of the discrete spectrum was proved in a bit more general form in a recent paper [3, theorem 4.6].

It turns out that the exponent $1 / 2$ in theorem 1 is sharp in the following sense.

Theorem 2. For arbitrary $\varepsilon>0$ and $-1<\nu<1$ there exists an operator $J \in$ $\mathcal{P}\left(\frac{1}{2}-\varepsilon\right)$ such that its discrete spectrum $\sigma_{d}(J)$ is infinite and, moreover, the set $E_{J}$ consists of a single point: $E_{J}=\{\nu\}$.

It is worth pointing out that for real Jacobi matrices $J$ with $b_{n}=\overline{b_{n}}, a_{n}=c_{n}>0$ the abovementioned problem is simple. As the discrete spectrum in this case is real, 
the only points \pm 1 (each one or both) can serve as its limit points. As is well known [4], the finiteness of the first moment

$$
\sum_{n} n\left\{\left|a_{n}-\frac{1}{2}\right|+\left|b_{n}\right|\right\}<\infty
$$

already guarantees the finiteness of the discrete spectrum of $J$.

The present paper appeared as an attempt to comprehend the remarkable Pavlov's paper $[1,2]$, which contains a beautiful synthesis of ideas from the operator theory and the function theory in the unit disk and which in our opinion was not fully appreciated by experts. The discrete model we are dealing with is comparatively simple that makes it possible to eliminate some inaccuracy and simplify the arguments by using the well developed techniques of perturbation determinants $[5,6]$ in Section 2. Section 3 follows the line of a seminal paper by L. Carleson [7] on zero sets of analytic functions in the unit disk which provides an analytic background for (1.5) and enables one to complete the proof of Theorem 1. In Section 4 we give some preliminary information about the scattering problem for real matrices, which is a key tool in the proof of Theorem 2]n Section 5. For the reader's convenience some results on perturbation determinants for complex Jacobi matrices are collected in Appendix.

Let us note in conclusion that the theory of complex Jacobi matrices has undergone a splash of activity lately in connection with the theory of formal orthogonal polynomials, Padé approximations and $J$-continued fractions [8-12].

\section{Perturbation determinants and Jost solution.}

In a recent paper [6] R. Killip and B. Simon applied the perturbation determinants technique to the spectral theory of real symmetric Jacobi matrices. Note that the most important results from [6, sect.2] remain valid for the complex matrices as well. For readers convenience we provide some information about perturbation determinants in Appendix.

Throughout the rest of the paper we assume the finiteness of the first moment

$$
\sum_{k=0}^{\infty}(k+1)\left\{\left|a_{k}-\frac{1}{2}\right|+\left|b_{k}\right|+\left|c_{k}-\frac{1}{2}\right|\right\}<\infty,
$$

although some results (in a weakened form) hold under less restrictive assumptions.

Under condition (2.1) the operator $J$ is a trace class perturbation of $J_{0}$, i.e., $\Delta J=J-J_{0} \in \mathcal{S}_{1}$, and so for $\lambda \notin[-1,1]$

$$
(J-\lambda)\left(J_{0}-\lambda\right)^{-1}=I+\Delta J\left(J_{0}-\lambda\right)^{-1},
$$

and thereby the perturbation determinant [5, Chap. IV.3]:

$$
\Delta(z, J):=\operatorname{det}(J-\lambda)\left(J_{0}-\lambda\right)^{-1} ; \quad \lambda(z)=\frac{z+z^{-1}}{2},
$$

is well defined (see, e.g., [5, chap. IV.3]).

The main properties of the perturbation determinant are displayed in the following statement $[5, \mathrm{p} .217 ; 6$, Theorem 2.5].

Proposition 2.1. Under assumption (2.1) the function $\Delta$ belongs to the disk algebra $\mathcal{A}$ of functions analytic in the unit disk $\mathbb{D}=\{z:|z|<1\}$ and continuous up to the unit circle. $\Delta\left(z_{0}\right)=0$ for $\left|z_{0}\right|<1$ if and only if $\lambda\left(z_{0}\right) \in \sigma_{d}(J)$, and the order of zero equals the algebraic multiplicity of the eigenvalue $\lambda\left(z_{0}\right)$. 
A key role in the theory is played by the following approximation relation for perturbation determinants. Denote by

$$
J_{m}=\left(\begin{array}{ccccc}
b_{0} & c_{0} & & & \\
a_{0} & b_{1} & c_{1} & & \\
& \ddots & \ddots & \ddots & \\
& & \ddots & \ddots & c_{m-1} \\
& & & a_{m-1} & b_{m}
\end{array}\right), \quad J_{0, m}=\left(\begin{array}{ccccc}
0 & \frac{1}{2} & & & \\
\frac{1}{2} & 0 & \frac{1}{2} & & \\
& \ddots & \ddots & \ddots & \\
& & \ddots & \ddots & \frac{1}{2} \\
& & & \frac{1}{2} & 0
\end{array}\right)
$$

the matrices of order $m+1$ obtained by taking the first $m+1$ rows and columns of $J J_{0}$, respectively. Then ([6, formula (2.59)])

$$
\Delta(z, J)=\lim _{m \rightarrow \infty} \operatorname{det}\left(\frac{J_{m}-\lambda}{J_{0, m}-\lambda}\right)
$$

uniformly on compact subsets of $\mathbb{D}$ (for the proof of (2.2) see Appendix).

Along with the initial matrices $J, J_{0}$ consider the associated matrices $J^{(n)}, J_{0}^{(n)}$, obtained by dropping the first $n+1$ rows and columns

$$
J^{(n)}=\left(\begin{array}{cccc}
b_{n+1} & c_{n+1} & & \\
a_{n+1} & b_{n+2} & c_{n+2} & \\
& \ddots & \ddots & \ddots
\end{array}\right), \quad J_{0}^{(n)}=J_{0},
$$

and $J^{(-1)}=J$. The matrices $J_{m}^{(n)}$ are defined in a natural way. By expanding the determinant $\operatorname{det}\left(J_{m}-\lambda\right)$ over the first row and $\operatorname{dividing}$ by $\operatorname{det}\left(J_{0, m}-\lambda\right)$ we obtain

$$
\begin{gathered}
\operatorname{det}\left(\frac{J_{m}-\lambda}{J_{0, m}-\lambda}\right)=\left(b_{0}-\lambda\right) \operatorname{det}\left(\frac{J_{m-1}^{(0)}-\lambda}{J_{0, m-1}-\lambda}\right) \operatorname{det}\left(\frac{J_{0, m-1}-\lambda}{J_{0, m}-\lambda}\right)- \\
-a_{0} c_{0} \operatorname{det}\left(\frac{J_{m-2}^{(1)}-\lambda}{J_{0, m-2}-\lambda}\right) \operatorname{det}\left(\frac{J_{0, m-2}-\lambda}{J_{0, m}-\lambda}\right) .
\end{gathered}
$$

Next, a direct calculation (cf. [6, formula (2.10)] shows that

$$
\lim _{m \rightarrow \infty} \operatorname{det}\left(\frac{J_{0, m-j}-\lambda}{J_{0, m}-\lambda}\right)=(-2 z)^{j}, \quad j \in \mathbb{N}=\{1,2, \ldots\}
$$

and so taking a limit in (2.3) as $m \rightarrow \infty$ with 2.2) in mind leads to

$$
\Delta(z, J)=\left(\lambda-b_{0}\right)\left(2 z \Delta\left(z, J^{(0)}\right)\right)-a_{0} c_{0}\left(4 z^{2} \Delta\left(z, J^{(1)}\right)\right) .
$$

Since (2.4) holds for any matrix $J$, write it for $J=J^{(n)}$ :

$$
\Delta\left(z, J^{(n)}\right)=\left(\lambda-b_{n+1}\right)\left(2 z \Delta\left(z, J^{(n+1)}\right)\right)-a_{n+1} c_{n+1}\left(4 z^{2} \Delta\left(z, J^{(n+2)}\right)\right) .
$$

Multiply (2.5) by $z^{n}$ and put $\psi_{n}:=z^{n} \Delta\left(z, J^{(n)}\right)$. After the shift of indices we have

$$
\psi_{m-1}(z)+2 b_{m} \psi_{m}(z)+4 a_{m} c_{m} \psi_{m+1}(z)=2 \lambda \psi_{m}(z), \quad m \geq 0 .
$$

Another important property of perturbation determinants is contained in the following result [6, proposition 2.14], its proof is given in Appendix. 
Proposition 2.2. Under condition (2.1) the relation

$$
\lim _{m \rightarrow \infty} \Delta\left(z, J^{(m)}\right)=1
$$

holds uniformly on the closed unit disk $\overline{\mathbb{D}}$.

Therefore for real symmetric $J$ the solution $\psi_{m}$ of (2.6) up to a constant factor (parameter) agrees with the Jost solution of the equation $(J-\lambda) \psi=0$ (see [13]).

In assumption (2.1) denote

$$
H(n):=\sum_{j=n}^{\infty}\left(\left|2 b_{m}\right|+\left|4 a_{m} c_{m}-1\right|\right), \quad H(n, m):=\prod_{j=n+1}^{n+m-1}(1+H(j)) .
$$

It is easy to see that $\{H(n)\} \in \ell^{1}$ and the sequences $H(\cdot)$ and $H(\cdot, m)$ decrease monotonically.

We can state the main result of the section as follows.

Theorem 2.3. Under (2.1) the Taylor coefficients of the perturbation determinant

$$
\Delta(z, J)=\sum_{j=0}^{\infty} \delta_{j} z^{j}
$$

admit the bound

$$
\left|\delta_{j}\right| \leq \prod_{j=1}^{\infty}(1+H(j)) \sum_{m=\left[\frac{j}{2}\right]}^{\infty}\left\{\left|2 b_{m}\right|+\left|4 a_{m} c_{m}-1\right|\right\},
$$

where $[x]$ is an integer part of $x$. In particular, $\Delta$ belongs to the space $W_{+}$of absolutely convergent Taylor series.

Proof. As a first step we show that $\psi_{n}$ obeys the "discrete integral" equation of Volterra type. To that end we introduce a function

$$
G(n, m, z)=\left\{\begin{array}{cl}
2 \frac{z^{m-n}-z^{n-m}}{z-z^{-1}}, & m \geq n, \\
0, & m<n,
\end{array}\right.
$$

for which

$$
\frac{1}{2} G(n, m+1)+\frac{1}{2} G(n, m-1)-\lambda G(n, m)=\delta(n, m)
$$

holds with $\delta(n, m)$ the Kronecker symbol. Now let us multiply (2.10) by $\psi_{m}$, (2.6) by $\frac{1}{2} G(n, m)$, subtract the latter from the former and sum up the equalities obtained over $m$ from $n$ to $N$. We have

$$
\begin{aligned}
& \psi_{n}=\sum_{m=n}^{N}\left\{-b_{m} G(n, m)+\left(\frac{1}{2}-2 a_{m-1} c_{m-1}\right) G(n, m-1)\right\} \psi_{m}+ \\
& +\frac{1}{2} G(n, N+1) \psi_{N}-2 a_{N} c_{N} G(n, N) \psi_{N+1} .
\end{aligned}
$$

By tending $N \rightarrow \infty$ and taking into account the explicit form of the kernel $G$ and (2.7) we see that

$$
\psi_{n}(z)=z^{n}+\sum_{m=n+1}^{\infty} M(n, m, z) \psi_{m}(z), \quad n=-1,0,1, \ldots
$$

where

$$
M(n, m, z)=-b_{m} G(n, m, z)+\left(\frac{1}{2}-2 a_{m-1} c_{m-1}\right) G(n, m-1, z),
$$


and $M(n, n, z)=0$. It is convenient to transform (2.11) by bringing in new variables

$$
\Delta\left(z, J^{(n)}\right)=z^{-n} \psi_{n}, \quad \hat{M}(n, m, z)=M(n, m, z) z^{m-n},
$$

and so

$$
\Delta\left(z, J^{(n)}\right)=1+\sum_{m=n+1}^{\infty} \hat{M}(n, m, z) \Delta\left(z, J^{(m)}\right)
$$

or otherwise,

$$
\Delta\left(z, J^{(n)}\right)-1=\sum_{m=n+1}^{\infty} \hat{M}(n, m, z)+\sum_{m=n+1}^{\infty} \hat{M}(n, m, z)\left(\Delta\left(z, J^{(m)}\right)-1\right) .
$$

It is clear that $\hat{M}$ is a polynomial on $z$, and as for $|z| \leq 1$

$$
\begin{gathered}
\left|G(n, m, z) z^{m-n}\right|=2\left|z^{2(m-n)}-1\right|\left|z-z^{-1}\right|^{-1} \leq 2|z||m-n|, \\
\left|G(n, m-1, z) z^{m-n}\right| \leq 2|z|^{2}|m-n-1|<2|z||m-n|,
\end{gathered}
$$

then

$$
|\hat{M}(n, m, z)| \leq|z||m-n|\left\{\left|2 b_{m}\right|+\left|1-4 a_{m-1} c_{m-1}\right|\right\}, \quad|z| \leq 1 .
$$

Condition (2.1) ensures the series

$$
g(n, z)=\sum_{m=n+1}^{\infty} \hat{M}(n, m, z)
$$

to converge absolutely and uniformly inside $\overline{\mathbb{D}}$ and by (2.13)

$$
|g(n, z)| \leq \sum_{m=n+1}^{\infty} m h_{m}, \quad h_{m}=\left|2 b_{m}\right|+\left|1-4 a_{m-1} c_{m-1}\right| .
$$

The standard method of succesive approximations (cf., e.g., [14, lemma 7.8]) gives

$$
\left|\Delta\left(z, J^{(n)}\right)-1\right| \leq \exp \left(\sum_{m=1}^{\infty} m h_{m}\right) \cdot \sum_{m=n+1}^{\infty} m h_{m}, \quad n \geq-1,|z| \leq 1 .
$$

Consider now the Taylor series expansion of the function $\Delta\left(z, J^{(n)}\right)$ :

$$
\Delta\left(z, J^{(n)}\right)=1+\sum_{j=1}^{\infty} \kappa(n, j) z^{j}
$$

and estimate its Taylor coefficients. By the Cauchy inequality

$$
|\kappa(n, j)| \leq C \sum_{m=n+1}^{\infty} m h_{m},
$$

where by $C$ we denote some positive constants which do not depend on the space variables and the spectral parameter. In particular, $\kappa(n, j) \rightarrow 0$ as $n \rightarrow \infty$ and fixed $j$.

The more accurate bound takes into account the dependence on the second index $j$. If we plug (2.14) in (2.5) and match the corresponding coefficients we have $(2.15)$

$$
\kappa(n, j+1)=\kappa(n+1, j-1)-\sum_{m=n+1}^{\infty}\left\{2 b_{m} \kappa(m, j)+\left(4 a_{m} c_{m}-1\right) \kappa(m+1, j-1)\right\}
$$


for $j \geq 2$,

$$
\kappa(n, 1)=-2 \sum_{m=n+1}^{\infty} b_{m}, \quad \kappa(n, 2)=-\sum_{m=n+1}^{\infty}\left\{2 b_{m} \kappa(m, 1)+\left(4 a_{m} c_{m}-1\right)\right\} .
$$

The induction on $j$ coupled with (2.15) leads to

$$
|\kappa(n, j)| \leq H(n, j) H\left(n+1+\left[\frac{j}{2}\right]\right), \quad n \geq-1,
$$

where $H(n), H(n, m)$ are taken from (2.8). Hence

$$
|\kappa(n, j)| \leq \prod_{j=1}^{\infty}(1+H(j)) \sum_{m=n+1+\left[\frac{j}{2}\right]}^{\infty}\left\{\left|2 b_{m}\right|+\left|4 a_{m} c_{m}-1\right|\right\} .
$$

The desired bound (2.9) is (2.16) with $n=-1$. The proof is complete.

Corollary 2.4. Let for $J$ (1.2) the moment of the order $n+1$ is finite

$$
M_{n+1}:=\sum_{k=0}^{\infty}(k+1)^{n+1}\left\{\left|a_{k}-\frac{1}{2}\right|+\left|b_{k}\right|+\left|c_{k}-\frac{1}{2}\right|\right\}<\infty .
$$

Then the $n$ 's derivative $\Delta^{(n)}(z)$ belongs to $W_{+}$and

$$
\max _{z \in \overline{\mathbb{D}}}\left|\Delta^{(n)}(z)\right| \leq C(J) \frac{4^{n}}{n+1} M_{n+1},
$$

where a positive constant $C(J)$ depends only on $J$.

Proof. The statement is a simple consequence of (2.17), the series expansion

$$
\Delta^{(n)}(z)=\sum_{j=0}^{\infty}(j+1) \ldots(j+n) \delta_{j+n} z^{j}
$$

and bounds (2.9).

\section{ZERO SETS FOR ClASSES OF ANALYTIC FUNCTIONS IN THE UNIT DISK.}

Let $X$ be a class of functions from the disk algebra $\mathcal{A}$. A closed point set $E$ on the unit circle $\mathbb{T}$ is called the set of uniqueness for $X$, if there is no nontrivial function $f \in X$, which vanishes on $E$. Otherwise $E$ is a zero set for $X$. When $X=\mathcal{A}$, then according to Fatou's theorem [15], a set $E$ is a zero set for $\mathcal{A}$ if and only if $E$ has the Lebesgue measure zero: $|E|=0$. If $X$ is a subclass of $\mathcal{A}$, the zero sets may possess some additional properties. The investigation of (metric) properties of zero sets goes back to A. Beurling [16] and L. Carleson [7] (see also [17] for the extension of these results). For instance let $\mathcal{A}_{\infty}$ be a set of all functions from $\mathcal{A}$ which have all their derivatives in the same class $\mathcal{A}$. A closed point set $E=\mathbb{T} \backslash \cup_{j} l_{j}$ with the adjacent $\operatorname{arcs} l_{j}$ is the zero set for $\mathcal{A}_{\infty}$ if and only if

$$
\sum_{j}\left|l_{j}\right| \log \frac{1}{\left|l_{j}\right|}<\infty \Leftrightarrow \int_{\mathbb{T}}|\log \rho(\zeta, E)| d m<\infty, \quad \rho(\zeta, E)=\operatorname{dist}(\zeta, E),
$$

where $d m$ is the normalized Lebesgue measure on $\mathbb{T}$. We are interested in certain subclasses of the class $\mathcal{A}_{\infty}$, and for zero sets some more stringent conditions than (3.1) emerges.

Let $g \in \mathcal{A}_{\infty}$

$$
G_{n}(g)=G_{n}:=\max _{z \in \overline{\mathbb{D}}}\left|g^{(n)}(z)\right|, \quad n \in \mathbb{Z}_{+} .
$$


The following result (Taylor's formula with the reminder term) can be easily proved by induction on $n$.

Lemma 3.1. Let $g \in \mathcal{A}_{\infty}$. Then for an arbitrary $n \in \mathbb{N}$ and points $z, w$ in $\overline{\mathbb{D}}$ the inequality holds

$$
\left|g(z)-\sum_{k=0}^{n-1} \frac{g^{(k)}(w)}{k !}(z-w)^{k}\right| \leq \frac{G_{n}}{n !}|z-w|^{n} .
$$

Consider the zero set of a function $g$ on $\mathbb{T}$ :

$$
F(g)=F:=\left\{\zeta \in \mathbb{T}: g^{(n)}(\zeta)=0 \text { for all } n \in \mathbb{Z}_{+}\right\} .
$$

Clearly $F$ is a closed point set of measure zero which can be identified with a point set on $[0,2 \pi)$. Let $F_{t}=\{x: \operatorname{dist}(x, F) \leq t\}$ and put $\phi_{F}(t):=\phi(t)=\left|F_{t}\right|$. The properties of $\phi$ as $t \rightarrow 0$ play a crucial role in the study of zero sets in some classes of functions in $\mathcal{A}_{\infty}$ with certain bounds on their derivatives. The function $\phi$ is also known as the distribution function of $d_{F}(x)=\operatorname{dist}(x, F), x \in \mathbb{R}$. By the change of variables formula for any measurable function $h$

$$
\int_{F_{s}} h\left(d_{F}(t)\right) d t=\int_{0}^{s} h(u) d \phi(u)
$$

holds. For a function $g \in \mathcal{A}_{\infty}$ we define the value (cf. [1])

$$
T(s):=\inf _{k \geq 0} \frac{G_{k}(g)}{k !} s^{k}, \quad s>0 .
$$

Lemma 3.2. Let $g \in \mathcal{A}_{\infty}$ and $g \neq 0$. Then

$$
\int_{0}^{2 \pi} \log T(s) d \phi(s)>-\infty .
$$

Proof. Put in (3.3) $w=\exp \left(i \theta_{0}\right), \theta_{0} \in F$ and $z=\exp (i \theta)$. Then

$$
\left|g\left(e^{i \theta}\right)\right| \leq \frac{G_{n}}{n !}\left|\theta-\theta_{0}\right|^{n} .
$$

The right hand side of this inequality contains two parameters $\left(\begin{array}{ll}n & \theta_{0}\end{array}\right)$. Taking the minimum we have

$$
\left|g\left(e^{i \theta}\right)\right| \leq \inf _{n \geq 0} \frac{G_{n}}{n !}\left(d_{F}(\theta)\right)^{n}=T\left(d_{F}(\theta)\right), \quad \log \left|g\left(e^{i \theta}\right)\right| \leq \log T\left(d_{F}(\theta)\right) .
$$

It remains only to apply (3.4) and the well- known property

$$
\int_{0}^{2 \pi} \log \left|g\left(e^{i \theta}\right)\right| d \theta>-\infty
$$

The lemma is proved.

Let us now introduce the main object related to the zeros of $g$ inside the disk:

$$
E(g)=E:=\left\{\zeta \in \mathbb{T}: \exists\left\{z_{n}\right\} \in \mathbb{D}, z_{n} \rightarrow \zeta, g\left(z_{n}\right)=0\right\} .
$$

Lemma 3.3. $E \subset F$.

Proof. Assume that $g^{(k)}(w)=0, k=0,1, \ldots, n-2$ and $g^{(n-1)}(w) \neq 0$ for some $w \in E$. By (3.3)

$$
\left|g(z)-\frac{g^{(n-1)}(w)}{(n-1) !}(z-w)^{n-1}\right| \leq \frac{G_{n}}{n !}|z-w|^{n} .
$$


Putting $z=z_{k}$ and tending $k \rightarrow \infty$ leads to the contradiction.

Proof of theorem 1 rests on Corollary 2.4. For matrices $J \in \mathcal{P}(\beta)$ (1.4) condition (2.17) holds a fortiori for all $n$, and it is easy to find bounds for the moments $M_{r}$. As

$$
\left|a_{k}-\frac{1}{2}\right|+\left|b_{k}\right|+\left|c_{k}-\frac{1}{2}\right| \leq C \exp \left(-C(k+1)^{\beta}\right), \quad 0<\beta<1,
$$

(by $C$ we denote positive constants which depend only on the original matrix $J$ ), we have

$$
M_{r} \leq C \sum_{k=0}^{\infty}(k+1)^{r} \exp \left(-\frac{C}{2}(k+1)^{\beta}\right) \exp \left(-\frac{C}{2}(k+1)^{\beta}\right) .
$$

An undergraduate analysis of the function $u(x)=x^{r} \exp \left(-\frac{C}{2} x^{\beta}\right)$ gives

$$
\max _{x \geq 0} u(x)=u\left(x_{0}\right)=\left(\frac{2 r}{C \beta}\right)^{\frac{r}{\beta}} e^{-\frac{r}{\beta}}=\left(\frac{2}{C \beta e}\right)^{\frac{r}{\beta}} r^{\frac{r}{\beta}}, \quad x_{0}=\left(\frac{2 r}{C \beta}\right)^{\frac{1}{\beta}},
$$

so that

$$
M_{r} \leq B\left(\frac{2}{C \beta e}\right)^{\frac{r}{\beta}} r^{\frac{r}{\beta}}, \quad B=C \sum_{k=0}^{\infty} \exp \left\{-\frac{C}{2}(k+1)^{\beta}\right\}
$$

Hence

$$
G_{n}(\Delta)=\max _{z \in \overline{\mathbb{D}}}\left|\Delta^{(n)}(z)\right| \leq C \frac{4^{n}}{n+1}\left(\frac{2}{C \beta e}\right)^{\frac{n+1}{\beta}}(n+1)^{\frac{n+1}{\beta}}, \quad n \geq 0 .
$$

The inequalities

$$
\left(\frac{n+1}{n}\right)^{\frac{n}{\beta}}<e^{\frac{1}{\beta}}, \quad(n+1)^{\frac{1}{\beta}}<e^{\frac{n+1}{\beta}}, \quad(n+1)^{\frac{n+1}{\beta}}<e^{\frac{1}{\beta}} n^{\frac{n}{\beta}} e^{\frac{n+1}{\beta}}
$$

lead to

$$
G_{n}(\Delta) \leq C C_{1}^{n} n^{\frac{n}{\beta}}, \quad n \geq 0 .
$$

In other words, the perturbation determinant for $J \in \mathcal{P}(\beta)$ belongs to the Gevre class $\mathcal{G}_{\beta}$.

Let us go over to the function $T$ (3.5). As $n !>n^{n} e^{-n}$, then

$$
T(s) \leq C \inf _{n}\left(C_{1} e s\right)^{n} n^{\frac{n}{\beta}-n}=C \inf _{n} t^{n} n^{\alpha n},
$$

where $t=C_{1}$ es $<1 / 2$ for small enough $s, \alpha=\beta^{-1}-1>0$. An elementary analysis of the function $v(x)=t^{x} x^{\alpha x}$ shows that

$$
\min _{x \geq 0} v(x)=v\left(x_{1}\right)=\exp \left(-\frac{\alpha}{e} t^{-\frac{1}{\alpha}}\right), \quad x_{1}=\frac{1}{e} t^{-\frac{1}{\alpha}} \gg 1 .
$$

Although the number $x_{1}$ is not in general an integer, by putting $n=\left[x_{1}\right]$, it is not hard to make sure that

$$
T(s) \leq C \exp \left\{-C\left(\frac{1}{s}\right)^{\frac{\beta}{1-\beta}}\right\}, \quad s \leq s_{0} .
$$

Applying lemma 3.2 we conclude that

$$
\int_{0} \frac{d \phi_{F}(s)}{s^{\frac{\beta}{1-\beta}}}<\infty, \quad F=F(\Delta) .
$$


Suppose first that $0<\beta<1 / 2$. It is proved in [7] that the convergence of integral (3.7) is equivalent to convergence of the series

$$
\sum_{j=1}^{\infty}\left|l_{j}\right|^{\frac{1-2 \beta}{1-\beta}}<\infty
$$

where $\left\{l_{j}\right\}$ are adjacent intervals of the closed point set $F$ (as a set on $[0,2 \pi)$ ), and thereby

$$
\tau(F) \leq \frac{1-2 \beta}{1-\beta}
$$

The inequality $\operatorname{dim} F \leq \tau(F)$ follows from the general theory of fractal dimension (see [18]). By lemma $3.3 E=E(\Delta) \subset F$, and so due to monotonicity of dimensions

$$
\operatorname{dim} E \leq \tau(E) \leq \frac{1-2 \beta}{1-\beta} .
$$

Inequality (1.5) stems out of an obvious relation between $E_{J} \quad E$.

Consider now the case $\beta=\frac{1}{2}$, so that

$$
\int_{0} \frac{d \phi_{F}(s)}{s}<\infty
$$

We show that the latter is impossible for nonempty $F$. Suppose that the adjacent arcs $l_{j}=\left(\alpha_{j}, \beta_{j}\right)$ are labelled in the order of decreasing length, i.e., $\left|l_{1}\right| \geq\left|l_{2}\right| \geq \ldots$. Let $0<t_{1}<t_{2}<\frac{1}{2}\left|l_{1}\right|=\frac{\beta_{1}-\alpha_{1}}{2}$. By the definition of $\phi$

$$
\phi\left(t_{2}\right)-\phi\left(t_{1}\right)=\left|\left\{x: t_{1}<\operatorname{dist}(x, F) \leq t_{2}\right\}\right| .
$$

For the interval $I\left(t_{1}, t_{2}\right)=\left(\alpha_{1}+t_{1}, \alpha_{1}+t_{2}\right)$ the relation

$$
I\left(t_{1}, t_{2}\right) \subset\left\{x: t_{1}<\operatorname{dist}(x, F) \leq t_{2}\right\}
$$

takes place, and so $\phi\left(t_{2}\right)-\phi\left(t_{1}\right) \geq t_{2}-t_{1}$. Hence the measure $d \phi_{F}$ dominates the Lebesgue measure and integral (3.8) diverges. So we arrive at the following conclusion: if $J \in \mathcal{P}\left(\frac{1}{2}\right)$, then $F$ (as well as $E$ ) is empty, which exactly means finiteness of the discrete spectrum $\sigma_{d}(J)$. The proof is complete.

Remark 1. Corollary 2.4 enables one to obtain some conditions on the "size" of the set $E_{J}$ in much more general situations. For instance, if $J$ satisfies (2.17), then $\Delta \in \mathcal{A}_{\infty}$, and so for the set $E(\Delta)$ (3.1) is true, and the same condition (taken over to the interval $[-2,2]$ ) holds for $E_{J}$. As another example, let us take the class of Jacobi matrices $J$ subject to

$$
\left|a_{k}-\frac{1}{2}\right|+\left|b_{k}\right|+\left|c_{k}-\frac{1}{2}\right| \leq C \exp \left\{-C(\log (k+1))^{\gamma}\right\}, \quad \gamma>1,
$$

which contains the class $\mathcal{P}(\beta)$. It is not hard to verify that for perturbation determinants of such matrices

$$
\max _{z \in \mathbb{D}}\left|\Delta^{(n)}(z)\right| \leq C \exp \left\{C n^{p}\right\}, \quad p=\frac{\gamma}{\gamma-1}>1,
$$

holds. The functions with such kind of bound for their derivatives were studied by B. Taylor and D. Williams in [19], wherein the condition on the zero sets

$$
\int_{\mathbb{T}}|\log \rho(\zeta, E)|^{q} d m<\infty, \quad \frac{1}{q}+\frac{1}{p}=1,
$$

was established. 
On the other hand, the finiteness of the discrete spectrum for $J \in \mathcal{P}\left(\frac{1}{2}\right)$ is caused by quasi-analyticity of the Gevre class $\mathcal{G}_{1 / 2}$, i.e., the lack of nontrivial function $f$, such that $f^{(n)}\left(\zeta_{0}\right)=0$ for all $n \geq 0$ and some $\zeta_{0} \in \mathbb{T}$. The well-known criterion of quasi-analyticity [20] makes it possible to prove slightly more general than (1.4) with $\beta=1 / 2$ results concerning the finiteness of the discrete spectrum.

Remark 2. The perturbation determinant of $J \in \mathcal{P}\left(\frac{1}{2}\right)$ may have boundary zeros on the unit circle, that can be observed on a simple example

$$
J: \quad a_{n}=c_{n}=\frac{1}{2}, \quad b_{0}=e^{i \theta}, \quad b_{1}=b_{2}=\ldots=0 .
$$

It is easy to compute $\Delta(z, J)=1-e^{i \theta} z$. These points (more precisely, their images under the Zhukovsky transformation) are known as the spectral singularities. In the above example with $\theta \neq 0, \pi$ the matrix $J$ has empty discrete spectrum and one spectral singularity at $\cos \theta \in(-1,1)$. Under condition (2.1) the spectral singularities form a closed set of measure zero. It is clear that $J \in \mathcal{P}\left(\frac{1}{2}\right)$ may have at most finite number of spectral singularities (cf. [3]). Indeed, otherwise we could find a point $\zeta_{0} \in \mathbb{T}$, for which $\Delta^{(n)}\left(\zeta_{0}, J\right)=0$ for all $n \geq 0$ (cf. (3.3)), that contradicts to quasi-analyticity of $\mathcal{G}_{1 / 2}$.

\section{ON SCATTERING DATA FOR REAL SYMMETRIC JACOBI MATRIX.}

In this section we study the relation between Fourier coefficients of the scattering function and the rate of stabilization of the Jacobi matrix entries in the case when both the discrete spectrum and resonance are absent. Our consideration is based on the scattering problem for semi-infinite real symmetric Jacobi matrix $[4,13,21]$. The main result of the section is lemma 4.2

Let

$$
J=\left(\begin{array}{ccccc}
b_{1} & a_{1} & & & \\
a_{1} & b_{2} & a_{2} & & \\
& \ddots & \ddots & \ddots & \\
& & \ddots & \ddots & \ddots
\end{array}\right)
$$

be a Jacobi matrix with the entries $a_{n}>0, b_{n}=\overline{b_{n}}$, subject to (1.5). Put $a_{0}=1$. It is well known (see $[4,13,21]$ ), that equation

$$
a_{n-1} y_{n-1}+b_{n} y_{n}+a_{n} y_{n+1}=\frac{1}{2}\left(z+z^{-1}\right) y_{n}, \quad z \in \overline{\mathbb{D}}, n \geq 1
$$

has a solution $f_{n}(z)$, called the Jost solution, with the following properties:

(i) $\lim _{n \rightarrow \infty} f_{n}(z) z^{-n}=1$ uniformly on $z \in \overline{\mathbb{D}}$;

(ii) for all $n \geq 0$ the function $f_{n}(z)$ belongs to the algebra $W_{+}$of absolutely convergent Taylor series, and $f_{n}(\bar{\zeta})=\overline{f_{n}(\zeta)}, \zeta \in \mathbb{T}$;

(iii) a function $f_{0}(z)$, known as the Jost function has at most finite number of zeros in $\overline{\mathbb{D}} \backslash\{ \pm 1\}$, all of them are real and simple, and their images in the $\lambda$-plane are points of the discrete spectrum of $J$;

(iv) two solutions $f_{n}(\zeta)$ and $\overline{f_{n}(\zeta)}$ of (4.2) are linearly independent for $\zeta \in \mathbb{T} \backslash\{ \pm 1\}$ and

$$
\langle f, \bar{f}\rangle=\frac{\zeta^{-1}-\zeta}{2},
$$

where $\langle f, \bar{f}\rangle:=a_{n-1}\left(f_{n-1} \overline{f_{n}}-f_{n} \overline{f_{n-1}}\right)$ is the Wronskian of these solutions. 
In view of (i) (ii) the following representation holds

$$
f_{n}(z)=\sum_{m=n}^{\infty} K(n, m) z^{m}, \quad z \in \overline{\mathbb{D}}, \quad n \in \mathbb{Z}_{+},
$$

where the entries $K(n, m)$ of the matrix $K$, called the transformation operator, are real and obey the relations $[14,22]$

$$
\begin{gathered}
a_{n}=\frac{K(n+1, n+1)}{2 K(n, n)}, \text { that is } K(n, n)=\prod_{j=n}^{\infty}\left(2 a_{j}\right)^{-1}, \\
b_{n}=\frac{K(n, n+1)}{2 K(n, n)}-\frac{K(n-1, n)}{2 K(n-1, n-1)}, \quad n \geq 1 .
\end{gathered}
$$

Note that the Jost function agrees (up to a constant factor which does not depend on the spectral parameter) with the perturbation determinant of $J$ (see, e.g., [6, formula (2.64)])

$$
f_{0}(z)=\left(\prod_{j=1}^{\infty} 2 a_{j}\right)^{-1} \Delta(z, J)
$$

Let $S(\zeta)=\overline{f_{0}(\zeta)}\left(f_{0}(\zeta)\right)^{-1}$ be the so called scattering function of the matrix $J, \zeta \in$ $\mathbb{T}$. As $f_{0}(\zeta) \neq 0, \zeta \in \mathbb{T}$ (under the assumption of the lack of resonance $f_{0}( \pm 1) \neq 0$ ), then $S(\zeta)$ is a continuous function on $\mathbb{T}$, and moreover $\overline{S(\zeta)}=S(\bar{\zeta})=S^{-1}(\zeta)$. Put

$$
F(n):=-\frac{1}{2 \pi} \int_{-\pi}^{\pi} S\left(e^{i \theta}\right) e^{i n \theta} d \theta .
$$

In the case when there is no discrete spectrum, the Fourier coefficients $F(n)$ and the matrix entries $K(n, m)$ of the transformation operator are connected by the equation of the inverse scattering problem (the Marchenko equation, see $[4,13]$ )

$$
\frac{\delta(n, m)}{K(n, n)}=K(n, m)+\sum_{l=n}^{\infty} K(n, l) F(l+m), \quad n, m \in \mathbb{Z}_{+} .
$$

As is known [14, formula (10.87)], under condition (1.5) the inequality

$$
\sum_{n=1}^{\infty} n|F(n+2)-F(n)|<\infty
$$

holds.

Consider the discrete analog of the Gelfand-Levitan operator $\mathcal{F}_{n}: \ell^{1}\left(\mathbb{Z}_{+}\right) \rightarrow$ $\ell^{1}\left(\mathbb{Z}_{+}\right)$

$$
\left(\mathcal{F}_{n} y\right)_{j}=\sum_{m=0}^{\infty} F(2 n+m+j) y_{m}, \quad j \in \mathbb{Z}_{+} .
$$

Here $n \geq 1$ is fixed.

Lemma 4.1. Under assumption $\sqrt{4.9}$ the operator $\mathcal{I}+\mathcal{F}_{n}$ is invertible in the space $\ell^{1}\left(\mathbb{Z}_{+}\right)$for all $n \geq 1$ and

$$
\left\|\left(\mathcal{I}+\mathcal{F}_{n}\right)^{-1}\right\| \rightarrow 1 \text { as } n \rightarrow \infty
$$


Proof. Put

$$
\hat{F}(n):=\sum_{j=0}^{\infty}|F(n+j)-F(n+j+2)|=\sum_{k=n}^{\infty}|F(k)-F(k+2)| .
$$

Since $F(n) \rightarrow 0$ as $n \rightarrow \infty$, then

$$
|F(k)| \leq \sum_{j=0}^{\infty}|F(k+2 j)-F(k+2 j+2)|,
$$

so that

$$
|F(k)|+|F(k+1)| \leq \hat{F}(k) .
$$

Hence $\{F(k)\} \in \ell^{1}\left(\mathbb{Z}_{+}\right)$, i.e., the operator $\mathcal{F}_{n}$ is compact. According to the Fredholm alternative, $\mathcal{I}+\mathcal{F}_{n}$ is invertible as soon as the equation

$$
\left(\mathcal{I}+\mathcal{F}_{n}\right) g=0
$$

has only trivial solution. As $S\left(e^{-i \theta}\right)=\overline{S\left(e^{i \theta}\right)}$, we have $F(n) \in \mathbb{R}$, and it suffices to restrict ourselves with the real solutions of (4.12). Let $g=\{g(k)\} \in \ell^{1}$ be such solution. Take the function

$$
\widetilde{g}(z):=\sum_{k=0}^{\infty} g(k) z^{k}
$$

Then

and so

$$
\begin{gathered}
(g, g)=\sum_{0}^{\infty}|g(k)|^{2}=\frac{1}{2 \pi} \int_{-\pi}^{\pi}\left|\widetilde{g}\left(e^{i \theta}\right)\right|^{2} d \theta \\
\left(\mathcal{F}_{n} g, g\right)=-\frac{1}{2 \pi} \int_{-\pi}^{\pi} S\left(e^{i \theta}\right) e^{2 i n \theta} \widetilde{g}^{2}\left(e^{i \theta}\right) d \theta
\end{gathered}
$$

$$
0=\left(\left(\mathcal{I}+\mathcal{F}_{n}\right) g, g\right)=\frac{1}{2 \pi} \int_{-\pi}^{\pi}\left(1-\Phi\left(e^{i \theta}\right)\right)\left|\widetilde{g}\left(e^{i \theta}\right)\right|^{2} d \theta
$$

where

$$
\Phi\left(e^{i \theta}\right)=S\left(e^{i \theta}\right) e^{2 i n \theta} \widetilde{g}^{2}\left(e^{i \theta}\right)\left|\widetilde{g}\left(e^{i \theta}\right)\right|^{-2} .
$$

Suppose that $\widetilde{g} \neq 0$. Since $\left|\Phi\left(e^{i \theta}\right)\right|=1$, then (4.13) implies $\Phi\left(e^{i \theta}\right) \equiv 1$, and so by the definition of the scattering function

$$
\frac{\widetilde{g}^{2}\left(e^{i \theta}\right)}{f_{0}^{2}\left(e^{i \theta}\right)} e^{2 i n \theta}=\frac{\left|\widetilde{g}\left(e^{i \theta}\right)\right|^{2}}{\left|f_{0}\left(e^{i \theta}\right)\right|^{2}} .
$$

Thereby for the function $h_{n}=\widetilde{g}^{2} f_{0}^{-2} e^{2 i n \theta}$ we have $h_{n}\left(e^{i \theta}\right)=\left|h_{n}\left(e^{i \theta}\right)\right|$. Thanks to the lack of the discrete spectrum and resonance, $f_{0}(z) \neq 0$ for $z \in \overline{\mathbb{D}}$, and hence $h_{n} \in W_{+}$. Then by the symmetry principle and the uniqueness theorem for analytic functions $h_{n} \equiv$ const in $\overline{\mathbb{D}}$. But for $n \geq 1 h_{n}(0)=0$, that is, $\tilde{g} \equiv 0$. The latter means that (4.12) has only trivial solution and so $\mathcal{I}+\mathcal{F}_{n}$ is invertible for each $n \geq 1$. The second statement is a simple consequence of inequalities

$$
\left\|\mathcal{F}_{n}\right\| \leq \sum_{j=0}^{\infty}|F(2 n+j)| \rightarrow 0, \quad n \rightarrow \infty
$$

and

$$
\left\|\left(\mathcal{I}+\mathcal{F}_{n}\right)^{-1}-\mathcal{I}\right\| \leq \frac{\left\|\mathcal{F}_{n}\right\|}{1-\left\|\mathcal{F}_{n}\right\|}
$$


The proof is complete.

Denote

$$
\tau(n, j)=K(n, n+j) K(n, n)-\delta(n, n+j) .
$$

Then (4.8) takes the form

$$
\tau(n, j)+\sum_{m=0}^{\infty} F(2 n+m+j) \tau(n, m)=-F(2 n+j), \quad j \geq 0,
$$

and hence

$$
\{\tau(n, j)\}_{j \geq 0}=-\left(\mathcal{I}+\mathcal{F}_{n}\right)^{-1}\{F(2 n+j)\}_{j \geq 0} .
$$

We need a slight modification of ([14, formula (10.110)]).

Lemma 4.2. Under assumption (4.9) the bound holds where $\hat{F}(n)$ is defined in 4.10 .

Proof. In the space $\ell^{1}\left(\mathbb{Z}_{+}\right)$consider the equation $\left(\mathcal{I}+\mathcal{F}_{n}\right) x=y$ or in other words

$$
x(j)+\sum_{m=0}^{\infty} F(2 n+j+m) x(m)=y(j), \quad x=\{x(j)\}, y=\{y(j)\}, j \geq 0 .
$$

Since $\mathcal{I}+\mathcal{F}_{n}$ is invertible for $n \geq 1$ then the solution (4.18) satisfies

$$
\|x\|_{1} \leq K\|y\|_{1}, \quad K=\sup _{n \geq 1}\left\|\left(\mathcal{I}+\mathcal{F}_{n}\right)^{-1}\right\|<\infty .
$$

By plugging (4.19) in (4.18), we obtain the bound for coordinates

$$
|x(j)| \leq \sup _{k \geq 2 n+j}|F(k)|\|x\|_{1}+|y(j)| \leq K \sup _{k \geq 2 n+j}|F(k)|\|y\|_{1}+|y(j)| .
$$

By (4.11) and on account of $\{\hat{F}(n)\}$ being non-increasing, we come to inequality

$$
|x(j)| \leq K \hat{F}(2 n+j)\|y\|_{1}+|y(j)| .
$$

We apply (4.20) twice. First, to equation (4.15) with

$$
x(j)=\tau(n, j), \quad y(j)=-F(2 n+j) .
$$

By (4.9)- (4.14)

$$
\left\|y_{n}\right\|_{1} \leq \sum_{k=0}^{\infty} \hat{F}(k)=C<\infty, \quad\left|y_{n}(j)\right| \leq \hat{F}(2 n+j),
$$

and therefore, 4.20) takes the form

$$
|\tau(n, j)| \leq C \hat{F}(2 n+j), \quad j \geq 0 .
$$

Next, write 4.15) with indices $n$ and $n+1$ and subtract the latter from the former:

$$
\left(\mathcal{I}+\mathcal{F}_{n}\right)\left(\tau_{n}-\tau_{n+1}\right)=y_{n}, \quad \tau_{n}=\{\tau(n, j)\}_{j \geq 0}, \quad y_{n}=\left\{y_{n}(j)\right\}_{j \geq 0},
$$

where

$$
y_{n}(j)=F(2 n+2+j)-F(2 n+j)+\sum_{m=0}^{\infty}\{F(2 n+j+m)-F(2 n+2+j+m)\} \tau(n+1, m) .
$$


It follows directly from (4.21) that

$$
\left.\left|y_{n}(j)\right| \leq|F(2 n+j)-F(2 n+2+j)|+C \hat{F}(2 n) \hat{(2 n}+j\right), \quad\left\|y_{n}\right\| \leq C \hat{F}(2 n),
$$

and so (4.20) implies

$$
|\tau(n, j)-\tau(n+1, j)| \leq \mid F(2 n+j)-F(2 n+2+j \mid+C \hat{F}(2 n) \hat{F}(2 n+1), \quad j \geq 0 .
$$

The desired bound (4.17) flows out of equalities (4.5), (4.6) and definition (4.14). Indeed, by 4.5)

$$
0<C_{1} \leq K^{2}(n, n) \leq C_{2}<\infty, \quad K(n, n) \rightarrow 1, \quad n \rightarrow \infty,
$$

and so by (4.22) with $j=0$

$$
\begin{gathered}
\left|2 a_{n}-1\right|=\frac{|K(n+1, n+1)-K(n, n)|}{K(n, n)} \leq C|\tau(n, 0)-\tau(n+1,0)| \leq \\
\leq C\left\{|F(2 n)-F(2 n+2)|+\hat{F}^{2}(2 n)\right\} .
\end{gathered}
$$

Next, since $|\tau(n, 1)| \leq C$ (see (4.21) , we have by (4.6)

$$
\left|2 b_{n}\right|=\frac{\left|4 a_{n-1}^{2} \tau(n-1,1)-\tau(n, 1)\right|}{K^{2}(n, n)} \leq C\left\{|\tau(n-1,1)-\tau(n, 1)|+\left|4 a_{n-1}^{2}-1\right|\right\} .
$$

Taking into account (4.22) for $j=1$, (4.23) and monotonicity of $\hat{F}(n)$ we see that $(4.24)\left|b_{n}\right| \leq C\{|F(2 n-1)-F(2 n+1)|+|F(2 n)-F(2 n+2)|+\hat{F}(2 n-2) \hat{F}(2 n-1)\}$.

Finally, (4.23) coupled with (4.24) gives (4.17), that completes the proof of lemma 4.2

\section{PAVlov's EXAmple.}

The proof of Theorem 2 is based on a specific "oscillatory integral", invented by Pavlov in $[2, \S 3]$

$$
V(z)=\int_{0}^{z} \exp \{-\chi(\xi)\} \cos (\gamma \chi(\xi)) d \xi
$$

where $\chi(\xi)=\frac{1}{32}\left(1+\xi^{2}\right)^{\gamma-1}$ and $\gamma$ is a small enough parameter, $0<\gamma<1$. The following properties of such integrals are of particular interest:

(i) $V \in \mathcal{A}_{\infty}$ and is analytic at every point $\zeta \in \mathbb{T} \backslash\{ \pm i\}$;

(ii) $V(0)=0,|V(z)| \leq 1$ for $z \in \overline{\mathbb{D}}$, and $V(z) \neq 0$ for $z \neq 0$;

(iii) $\operatorname{Im} V(z) \operatorname{Im} z>0$ for $z \notin \mathbb{R}$;

(iv) There is a sequence of points on the imaginary axis $z_{k}=i \Im z_{k}, 0<\Im z_{k}<1$, such that $\Im z_{k} \uparrow 1$ and $V\left(z_{k}\right)=V(i)$. Moreover $V\left(\bar{z}_{k}\right)=V\left(-z_{k}\right)=\overline{V(i)}$.

We will turn to some other properties of Pavlov's function in due course. Put

$$
f(\lambda)=-\frac{1}{V(-z(\lambda))}, \quad \lambda(z)=\frac{1}{2}\left(z+\frac{1}{z}\right) .
$$

It is clear that $f$ is analytic in $\{\operatorname{Im} \lambda>0\}$ and continuous (in fact infinitely differentiable) in $\{\operatorname{Im} \lambda \geq 0\}$ with $\operatorname{Im} f(x)=0$ for $x \in(-\infty,-1] \cup[1, \infty)$ and $\operatorname{Im} f(x)>0$ for $\lambda \in\{\operatorname{Im} \lambda>0\} \cup(-1,1)$. Hence,

$$
f(\lambda)=\alpha \lambda+\beta+\int_{-1}^{1} \frac{\operatorname{Im} f(x)}{x-\lambda} d x, \quad \alpha \geq 0, \beta=\bar{\beta}
$$


A coefficient $\alpha$ can be found from the limit relation

$$
\alpha=\lim _{y \rightarrow+\infty} \frac{f(i y)}{i y}=-\lim _{t \downarrow 0} \frac{2}{i\left(t^{-1}-t\right) V(i t)},
$$

and since $V(i t)=V^{\prime}(0) i t+O\left(t^{2}\right)$ for $t \rightarrow 0$, then

$$
\alpha=\frac{2}{V^{\prime}(0)}=\frac{2 e^{1 / 32}}{\cos \gamma}>0
$$

Define

$$
\widetilde{m}(\lambda):=A \int_{-1}^{1} \frac{\operatorname{Im} f(x)}{x-\lambda} d x=A(f(\lambda)-\alpha \lambda-\beta), \quad A^{-1}:=\int_{-1}^{1} \operatorname{Im} f(x) d x .
$$

The function $\widetilde{m}(\lambda)$ is the Weyl function for a certain Jacobi matrix

$$
\tilde{J}=\left(\begin{array}{cccc}
b_{1} & a_{1} & & \\
a_{1} & b_{2} & a_{2} & \\
& \ddots & \ddots & \ddots
\end{array}\right), \quad a_{j}>0, b_{j}=\overline{b_{j}} .
$$

Finally, put

$$
a_{0}^{2}:=-\frac{1}{\alpha A}, \quad b_{0}:=-\frac{\beta}{\alpha}-\frac{1}{\alpha \overline{V(i)}}, \quad a_{0}, b_{0} \in \mathbb{C},
$$

where $\alpha$ and $\beta$ are taken from representation (5.2), and construct a complex Jacobi matrix

$$
J=\left(\begin{array}{cc}
b_{0} & a_{0} \\
a_{0} & \tilde{J}
\end{array}\right) .
$$

Our goal is to prove that $J$ satisfies theorem 2 with $\nu=0 .{ }^{1}$

Let us begin with the discrete spectrum of $J$. Denote by $R(\lambda)=(J-\lambda)^{-1}$ the resolvent of $J$ and let $m(\lambda)=\left(R(\lambda) e_{0}, e_{0}\right)$ be its Weyl function. It is known [23], that

$$
m(\lambda)=\frac{1}{\lambda-b_{0}-a_{0}^{2} \widetilde{m}(\lambda)},
$$

and due to the choice of $a_{0}, b_{0}$ (5.4) and the definition of $\widetilde{m}$ we have

$$
\begin{aligned}
\lambda-a_{0}^{2} \widetilde{m}(\lambda)-b_{0}=\lambda+\frac{\widetilde{m}(\lambda)}{\alpha A}+\frac{\beta}{\alpha}+\frac{1}{\alpha \overline{V(i)}}= \\
=\frac{1}{\alpha}\left(f(\lambda)+\frac{1}{\overline{V(i)}}\right)=\frac{1}{\alpha}\left(\frac{1}{\overline{V(i)}}-\frac{1}{V(-z(\lambda))}\right) .
\end{aligned}
$$

Let $\lambda_{k}=\lambda\left(z_{k}\right)=\frac{1}{2}\left(z_{k}+z_{k}^{-1}\right)$, where $z_{k}$ is taken from (iv), and so $\lambda_{k} \in i \mathbb{R}_{-}$, $\lambda_{k} \rightarrow 0$ as $k \rightarrow \infty$. The property (iv) of $V$ yields $\lambda_{k}-b_{0}-a_{0}^{2} \widetilde{m}\left(\lambda_{k}\right)=0$, that is, $m$ has poles at $\lambda_{k}$, and hence $\left\{\lambda_{k}\right\} \in \sigma_{d}(J)$ and 0 is a limit point of $\sigma_{d}(J)$.

We want to show that there are no other limit points. The argument rests on the following result of the operator theory.

Lemma 5.1. Let $T$ be a bounded operator in the Hilbert space $\mathcal{H}$, such that $T$ and $T^{*}$ have common cyclic vector $h . A$ point $\lambda \in \mathbb{C}$ is an isolated point of the spectrum $T$ if and only if $\lambda$ is a singular point of the Weyl function $m(\lambda)=\left(R(\lambda) e_{0}, e_{0}\right)$, $R(\lambda)=(T-\lambda)^{-1}$.

\footnotetext{
${ }^{1}$ At the final stage of the proof we show that the general case only insignificantly differs from this particular one.
} 
Proof. We have to check that an isolated singularity of the resolvent $R(\lambda)$ is an unremovable singularity of the Weyl function(the inverse is obvious). Assume that $m$ is analytic at $\lambda$. As

$$
\left(R(\mu) T^{k} h, h\right)=\left(R(\mu)\left(T^{k}-\mu^{k}\right) h, h\right)+\mu^{k} m(\mu)=P(\mu)+\mu^{k} m(\mu),
$$

where $P$ is a polynomial, then the functions $\left(R(\mu) g_{n}, h\right)$ with $g_{n}=\sum_{k=0}^{n} c_{k} T^{k} h$, are also analytic at $\lambda$. By the premises the system $\left\{T^{k} h\right\}_{k \geq 0}$ is complete in $\mathcal{H}$, so that for an arbitrary $g \in \mathcal{H}$ there is a sequence $g_{n} \rightarrow g$ as $n \rightarrow \infty$. For small enough $\varepsilon>0$ a punctured disk $\{\mu: 0<|\mu-\lambda| \leq \varepsilon\}$ lies entirely in the resolvent set of $T$, i.e., $\|R(\mu)\| \leq C$ for $|\mu-\lambda|=\varepsilon$. Hence $\left(R(\mu) g_{n}, e_{0}\right)$ converges to $\left(R(\mu) g, e_{0}\right)$ uniformly in that disk and so $\left(R(\mu) g, e_{0}\right)$ is analytic at $\lambda$. Similarly by using the completeness of $\left\{\left(T^{*}\right)^{k} h\right\}_{k \geq 0}$, we can ascertain that $(R(\mu) g, f)$ is analytic at $\lambda$ for all $g, f \in \mathcal{H}$. It remains only to note that the weak analyticity implies the strong one (see, e.g., [24, Chapter V.3]), that is, $R$ is analytic at $\lambda$, as needed.

Assume now that there is a sequence $\left\{\lambda_{k}^{\prime}\right\} \in \sigma_{d}(J)$ and $\lambda^{\prime}=\lim _{k \rightarrow \infty} \lambda_{k}^{\prime} \in$ $[-1,1] \backslash 0$. By lemma 5.1] the Weyl function $m$ has poles at $\lambda_{k}^{\prime}$ and in view of (5.4), (5.5) $V\left(-z\left(\lambda_{k}^{\prime}\right)\right)=\overline{V(i)}$. But $-\lim _{k \rightarrow \infty} z\left(\lambda_{k}^{\prime}\right)=y^{\prime} \in \mathbb{T} \backslash\{ \pm i\}$ and since $V$ is analytic at $y^{\prime}$, then $V \equiv \overline{V(i)}$, that contradicts the definition of Pavlov's function $V$ (5.1).

The main problem we have to cope with is to show that $J$ belongs to $\mathcal{P}\left(\frac{1}{2}-\varepsilon\right)$. As a first step in that direction we find some preliminary rate of stabilization of the parameters $\left\{a_{n}, b_{n}\right\}$, which are the three term recurrence coefficients for orthonormal polynomilas with respect to the weight $w(x)=A \operatorname{Im} f(x)$ on $[-1,1]$ with $A$ defined in (5.3). As Pavlov's function $V$ is analytic at 1 and $V(1)>0$, $V^{\prime}(1)>0$, then

$$
\operatorname{Im} V\left(e^{i \theta}\right)=V^{\prime}(1) \sin \theta+O\left(\sin ^{2} \theta\right)
$$

for $\theta$ near $0, \pi$, and

$$
\frac{w(\cos \theta)}{\sin \theta}=\frac{\operatorname{Im} V\left(e^{i \theta}\right)}{\sin \theta\left|V\left(e^{i \theta}\right)\right|^{2}}, \quad 0 \leq \theta \leq \pi,
$$

is $2 \pi$-periodic and infinitely differentiable. Therefore for its Fourier coefficients

$$
\frac{w(\cos \theta)}{\sin \theta}=\sum_{n=-\infty}^{\infty} q_{n} e^{i n \theta}, \quad \sum_{n=1}^{\infty} n^{k}\left|q_{n}\right|<\infty
$$

holds for any positive integer $k$. According to [25, theorem 1$]$ the similar inequality is true for $\left\{a_{n}, b_{n}\right\}$ :

$$
\sum_{n=1}^{\infty} n^{k}\left\{\left|a_{n}-\frac{1}{2}\right|+\left|b_{n}\right|\right\}<\infty, \quad k=1,2, \ldots
$$

Relations (5.7) make it possible to invoke the scattering problem technique developed in the previous section. Note that we do not just pay a tribute to tradition, but apply an adequate machinery which produces the sharp rate of decay of $a_{n}-\frac{1}{2}$ $b_{n}$ for weights, related to Pavlov's function. We do hope that some contemporary methods (such as, e.g., the Riemann-Hilbert problem) will provide the solution of 
the problem we mention for more general classes of weights as efficient as it is done for the weights analytic in a neighborhood of $[-1,1]^{2}$ (see [26]).

Let $\left\{\tilde{f}_{n}\right\}_{n \geq 0}$ be the Jost solution of the equation $(\tilde{J}-\lambda) y=0$. It agrees up to a multiplicative factor which depends on the spectral parameter, with the Weyl solution for the same equation and so

$$
\tilde{M}(z):=-\tilde{m}(\lambda(z))=\frac{\tilde{f}_{1}(z)}{\tilde{f}_{0}(z)} .
$$

By using expression (4.3) for the Wronskian $\langle\tilde{f}, \bar{f}\rangle$ we obtain

$$
\left|\tilde{f}_{0}\left(e^{i \theta}\right)\right|^{2}=\left|\frac{\sin \theta}{\operatorname{Im} \tilde{M}\left(e^{i \theta}\right)}\right| \text {. }
$$

Let us make sure first that there are no resonance at the edges of continuous spectrum of $\tilde{J}$. Assume on the contrary that $\tilde{f}_{0}( \pm 1)=0$ at least for one choice of the sign. Since $A^{-1} \tilde{m}(\lambda)=-V^{-1}(-z(\lambda))-\alpha \lambda-\beta$ is bounded at some neighborhood of $\lambda= \pm 1$, it follows from (5.8) that $\tilde{f}_{1}( \pm 1)=0$, and hence by the recurrence relation (4.2) $\tilde{f}_{n}( \pm 1)=0$ that contradicts the asymptotic behaviour of the Jost solution.

In addition to the properties (i) - (iv) of Pavlov's function mentioned above, in $[2$, formula (3.1)] the bounds for its derivatives are found

$$
\left|V^{(r)}\left(e^{i \theta}\right)\right| \leq K^{r} r ! r^{\frac{r}{\delta}}, \quad \delta=1-\gamma, \quad r=0,1,2, \ldots,
$$

where $K=K(\gamma)$ is some positive constant. We show that the scattering function $\tilde{S}=\overline{\tilde{f}_{0}} \tilde{f}_{0}^{-1}$ obeys the same bounds with, perhaps, another constant $K$. By $[2$, lemma $3 . .1$ and remark], when a function $v$ satisfies (5.10) and functions $u$ and $u_{1}$ are analytic in a neighborhood of the image of $v$ and the closed unit disk $\overline{\mathbb{D}}$, respectively, then $u(v)$ and $u_{1} v$ satisfy (5.10) (with another constant $K$ ). That is why the Weyl function

$$
\tilde{M}(z)=A\left(\frac{1}{V(-z)}+\frac{\alpha}{2}\left(z+z^{-1}\right)+\beta\right)
$$

is subject to (5.10) $\left(V\left(e^{i \theta}\right) \neq 0\right)$. Next, since by (5.6)

$$
\left|\frac{\sin \theta}{\operatorname{Im} \tilde{M}\left(e^{i \theta}\right)}\right| \geq C>0
$$

for all $\theta$, then by using (5.9) we have

$$
\left|\left[\log \left|\tilde{f}_{0}\left(e^{i \theta}\right)\right|\right]^{(r)}\right| \leq K_{1}^{r} r ! r^{\frac{r}{\delta}}, \quad K_{1}=K_{1}(\gamma)>0, \quad r \in \mathbb{Z}_{+} .
$$

For the scattering function $\tilde{S}$ the equality

$$
\tilde{S}\left(e^{i \theta}\right)=\exp \left(-2 i \arg \tilde{f}_{0}\left(e^{i \theta}\right)\right)=\exp \left(-2 i H\left(\log \left|\tilde{f}_{0}\right|\right)\left(e^{i \theta}\right)\right),
$$

holds and it remains to verify that the class [5.10) is invariant under the Hilbert operator $H$.

\footnotetext{
${ }^{2}$ Our weight $w$ has one singular point at the origin.
} 
Lemma 5.2. Let $g$ be a $2 \pi$-periodic and infinitely differentiable function. Then

$$
\left\|(H g)^{(n)}\right\|_{\infty} \leq C\left(\left\|g^{(n)}\right\|_{\infty}+\left\|g^{(n+1)}\right\|_{\infty}\right),
$$

where $C$ is a universal constant and the Hilbert operator is defined by

$$
(H g)(\theta)=\frac{1}{\pi} \int_{0}^{\pi} \frac{g(\theta-t)-g(\theta+t)}{2 \tan \frac{t}{2}} d t .
$$

Proof. In the premises of lemma the integral (5.11) converges absolutely and the operator $H$ commutes with the differentiation $(H f)^{\prime}=H f^{\prime}$. Next, by the integration by parts in the partial Fourier sum and taking a limit we come to the following representation for $f$

$$
f(x)=\frac{1}{\pi} \int_{-\pi}^{\pi} f(x) d x+\frac{1}{\pi} \int_{-\pi}^{\pi} \phi(t) f^{\prime}(x+t) d t,
$$

where

Based on this equality we derive

$$
\phi(t)=\left\{\begin{array}{lc}
\frac{t-\pi}{2}, & 0<t \leq \pi \\
\frac{t+\pi}{2}, & -\pi \leq t<0 .
\end{array}\right.
$$

$$
\|f\|_{\infty} \leq C\left(\|f\|_{2}+\left\|f^{\prime}\right\|_{2}\right) .
$$

Since the Hilbert operator is unitary in $L^{2}(\mathbb{T})$, we have

$$
\|H f\|_{\infty} \leq C\left(\|f\|_{2}+\left\|f^{\prime}\right\|_{2}\right) \leq C\left(\|f\|_{\infty}+\left\|f^{\prime}\right\|_{\infty}\right),
$$

as was to be proved.

Thereby for the scattering function we see that

$$
\left|\tilde{S}^{(r-1)}\left(e^{i \theta}\right)\right| \leq K_{2}^{r} r ! r^{\frac{r}{\delta}}, \quad K_{2}=K_{2}(\gamma)>0, \quad r=1,2, \ldots
$$

With lemma 4.2 under the belt we can go over to matrix entries $a_{n}, b_{n}$. Let us integrate by parts (4.7) $r$ times bearing in mind that $\tilde{S}$ is a periodic function. Then

$$
\begin{gathered}
n^{r}(|F(2 n+2)-F(2 n)|+|F(2 n-1)-F(2 n+1)|) \leq \\
\leq \frac{1}{2 \pi 2^{r}} \int_{0}^{2 \pi}\left|\frac{d^{r}}{d \theta^{r}}\left[\tilde{S}\left(e^{i \theta}\right)\left(e^{2 i \theta}-1\right)\right]\right| d \theta .
\end{gathered}
$$

The multiplication on a regular function does not alter the structure of bounds (5.12), and so with some positive constant $K_{3}$

$|F(2 n+2)-F(2 n)|+|F(2 n-1)-F(2 n+1)| \leq C K_{3}^{r} r ! r^{\frac{r}{\delta}} n^{-r+1} \leq C n \inf _{r \geq 3} t^{r} r^{\alpha r}$

with $t=K_{3} n^{-1} \ll 1$ and $\alpha=1+\frac{1}{\delta}$. We can apply (3.6) to the right hand side of (5.13). For large enough $n$ we have

$$
|F(2 n+2)-F(2 n)|+|F(2 n-1)-F(2 n+1)| \leq C \exp \left(-C_{1} n^{\frac{\delta}{1+\delta}}\right),
$$

where

$$
C_{1}=\frac{1+\delta}{2 \delta} K_{3}^{-\frac{\delta}{1+\delta}}>0
$$

From (4.10) and (5.14) it follows that

$$
\hat{F}^{2}(2 n-2) \leq C \exp \left(-C_{1} n^{\frac{\delta}{1+\delta}}\right) \sum_{k=n}^{\infty} \exp \left(-C_{1} k^{\frac{\delta}{1+\delta}}\right) \leq C_{2} \exp \left(-C_{1} n^{\frac{\delta}{1+\delta}}\right),
$$


and by lemma 4.2

$$
\left|a_{n}-\frac{1}{2}\right|+\left|b_{n}\right| \leq C_{3} \exp \left(-C_{4} n^{\frac{\delta}{1+\delta}}\right)=C_{3} \exp \left(-C_{4} n^{\frac{1-\gamma}{2-\gamma}}\right), \quad C_{3}, C_{4}>0 .
$$

Given $\varepsilon>0$, we choose $\gamma$ so small that

$$
\frac{1-\gamma}{2-\gamma}>\frac{1}{2}-\varepsilon
$$

which means exactly $\tilde{J} \in \mathcal{P}(1 / 2-\varepsilon)$. Theorem [2] is now proved for $\nu=0$.

The general case can be treated by the same reasoning by using a modified Pavlov's function. Indeed, let $-1<\kappa<1$. Consider the function

$$
V_{\kappa}(z)=V\left(\frac{z-\kappa}{1-\kappa z}\right), \quad\left(V_{0}(z)=V(z)\right) .
$$

It satisfies (i)-(iv) with the only difference that its singular points on the unit circle $\mathbb{T}$ are $w_{\kappa}$ and $\bar{w}_{\kappa}$, where $w_{\kappa}=(i+\kappa)(1+i \kappa)^{-1}$. When $\kappa$ passes through $(-1,1)$ the point $w_{\kappa}$ describes the upper semicircle. Hence under an appropriate choice of $\kappa$ we can end up with any point $\nu,-1<\nu<1$ of accumulation for the discrete spectrum of $J \in \mathcal{P}\left(\frac{1}{2}-\varepsilon\right)$. Now theorem 2 is proved completely.

\section{APPENDIX.}

We prove here two results concerning perturbation determinants of complex Jacobi matrices following the line of [6, section 2], wherein they are established for real symmetric matrices.

$1^{0}$. Proof of proposition 2.2. The basic properties of Schatten-von Neumann operator ideals $\mathcal{S}_{p}$ and infinite determinants are presumed to be known (see, e.g., [5, Chapters III, IV]). The proof is broken up into steps.

1. Let

$$
R_{0}(z)=\left(J_{0}-\lambda(z)\right)^{-1}, \quad R_{0}(z)=\left\{r_{n m}(z)\right\}_{0}^{\infty}, \quad|z|<1,
$$

be the resolvent of the discrete laplacian. Its matrix entries can be found by direct computation

$$
r_{n m}(z)=\frac{z^{|n-m|}-z^{n+m+2}}{z-z^{-1}}=-\sum_{j=0}^{\min (n, m)} z^{1+|n-m|+2 j}, \quad n, m \geq 0, \quad|z|<1 .
$$

It is clear that

$$
\left|r_{n m}(z)\right| \leq \min (n, m)+1 .
$$

Take $\zeta \in \mathbb{T}$. The boundary value $r_{n m}(\zeta)$ exists for trivial reasons, however the matrix $\left\{r_{n m}(\zeta)\right\}$ is defined only formally (it does not correspond in general to a bounded operator in $\ell^{2}$ ). The situation can be revised by means of edging $R_{0}$ with appropriate diagonal matrices. Let

$$
D=\operatorname{diag}\left(d_{0}, d_{1}, \ldots\right), \quad d_{j} \geq 0, \quad \sum_{j} j d_{j}<\infty .
$$

Consider an operator function

$$
B(z):=D^{1 / 2} R_{0}(z) D^{1 / 2}=\left\{B_{n m}(z)\right\}_{0}^{\infty}, \quad|z|<1 .
$$


As $D^{1 / 2} \in \mathcal{S}_{2}$ (the Hilbert-Schmidt operator), then $B(z) \in \mathcal{S}_{2}$ (as a matter of fact, $B \in \mathcal{S}_{1}$, as a product of two operators from $S_{2}$, the fact we will make use of later on). Moreover, by (6.1)

$\|B(z)\|_{2}^{2}=\sum_{n, m}\left|B_{n m}(z)\right|^{2} \leq \sum_{n, m}\left(d_{m}^{1 / 2} d_{n}^{1 / 2}[\min (n, m)+1]\right)^{2} \leq\left(\sum_{j}(j+1) d_{j}\right)^{2}$,

and hence the boundary values $B(\zeta)=\lim _{z \rightarrow \zeta} B(z)$ belong to $\mathcal{S}_{2}$ and obeys (6.2). Finally,

$$
\|B(z)-B(\zeta)\|_{2}^{2}=\sum_{n, m}\left|B_{n m}(z)-B_{n m}(\zeta)\right|^{2} \rightarrow 0, \quad z \rightarrow \zeta
$$

by the Lebesgue dominated convergence theorem, and so $B(z)$ belongs to the disk algebra $\mathcal{A}$ (as an operator valued function in $\mathcal{S}_{2}$ ).

Let now $J$ be the Jacobi matrix (1.2), subject to (1.3). Put $d_{0}=\max \left(\left|b_{0}\right|,\left|c_{0}-\frac{1}{2}\right|\right)$, $d_{n}=\max \left(\left|a_{n-1}-\frac{1}{2}\right|,\left|b_{n}\right|,\left|c_{n}-\frac{1}{2}\right|\right), n \geq 1 \quad D=\operatorname{diag}\left(d_{0}, d_{1}, \ldots\right)$. The perturbation $\Delta J=J-J_{0}$ admits factorization $\Delta J=D^{1 / 2} U D^{1 / 2}$, where

$U=\left\{u_{n m}\right\}, \quad u_{n m}=\left\{\begin{array}{cc}\frac{a_{n-1}-\frac{1}{2}}{\sqrt{d_{n-1} d_{n}}}, & m=n-1, \\ \frac{b_{n}}{d_{n}}, & m=n, \\ \frac{c_{n}-\frac{1}{2}}{\sqrt{d_{n+1} d_{n}}}, & m=n+1,\end{array} \quad u_{n m}=0, \quad|n-m| \geq 2\right.$,

where we agree that $\frac{0}{0}=1$. Obviously, $\left|u_{n m}\right| \leq 1$, so that $\|U\| \leq 3$.

2. The function $t(z):=\operatorname{tr}\left(\Delta J R_{0}(z)\right)$ is well defined for $|z|<1$ under the condition $\Delta J \in \mathcal{S}_{1}$. Let us show that (2.1) yields $t \in \mathcal{A}$, and find the upper bound for this function. By the definition of the trace $t=t_{1}+t_{2}+t_{3}$, where

$t_{1}(z)=\sum_{0}^{\infty} b_{j} r_{j j}(z) ; t_{2}(z)=\sum_{0}^{\infty}\left(a_{j}-\frac{1}{2}\right) r_{j, j+1}(z) ; t_{3}(z)=\sum_{0}^{\infty}\left(c_{j}-\frac{1}{2}\right) r_{j+1, j}(z)$.

Inequality (6.1) along with condition (2.1) enables one to conclude that $t_{l} \in \mathcal{A}$, $l=1,2,3$ and

$$
|t(z)| \leq \sum_{0}^{\infty}(j+2)\left\{\left|a_{j}-\frac{1}{2}\right|+\left|b_{j}\right|+\left|c_{j}-\frac{1}{2}\right|\right\} .
$$

3. Write the perturbation determinant $\Delta$ with factorization of $\Delta J$ in mind

$$
\Delta(z, J)=\operatorname{det}\left(I+\Delta J R_{0}\right)=\operatorname{det}\left(I+D^{1 / 2} U D^{1 / 2} R_{0}\right)=\operatorname{det}(I+U B(z))
$$

(we use here the property $\operatorname{det}(I+A B)=\operatorname{det}(I+B A)$ ). As $B(z)$ is a nice function taking the values from $\mathcal{S}_{2}$, it looks instructive to go over to regularized determinants [5, Chapter IV, §2]:

$$
\Delta(z, J)=\operatorname{det}_{2}(I+U B(z)) e^{\operatorname{tr}\left(\Delta J R_{0}(z)\right)} .
$$

In view of $\operatorname{det}_{2}$ being continuous on the operator argument [4, theorem IV.2.1] and $t$ being continuous in $\overline{\mathbb{D}}$, (6.4) implies $\Delta \in \mathcal{A}$.

Apply now (6.4) for the associated matrix $J^{(k)}$

$$
\Delta\left(z, J^{(k)}\right)=\operatorname{det}_{2}\left(I+U_{k} B_{k}(z)\right) e^{\operatorname{tr}\left(\Delta J^{(k)} R_{0}(z)\right)} .
$$


The bordering matrix $D_{k}$ takes the form $D_{k}=\operatorname{diag}\left(d_{0}^{(k)}, d_{1}^{(k)}, \ldots\right), d_{j}^{(k)}=d_{j+k}$. By (6.2)

$$
\left\|B_{k}(z)\right\|_{2}^{2} \leq\left(\sum_{j}(j+1) d_{j}^{(k)}\right)^{2}
$$

and the Lebesgue theorem provides $\lim _{k \rightarrow \infty}\left\|B_{k}(z)\right\|_{2}=0$ uniformly in $\overline{\mathbb{D}}$, whence it follows $\lim _{k \rightarrow \infty} \operatorname{det}_{2}\left(I+U_{k} B_{k}(z)\right)=1$. By (6.3)

$$
\left|t^{(k)}(z)\right|=\left|\operatorname{tr}\left(\Delta J^{(k)} R_{0}(z)\right)\right| \leq \sum_{0}^{\infty}(j+2)\left\{\left|a_{j+k}-\frac{1}{2}\right|+\left|b_{j+k}\right|+\left|c_{j+k}-\frac{1}{2}\right|\right\},
$$

so that $\lim _{k \rightarrow \infty}\left|t^{(k)}(z)\right|=0$ uniformly in $\overline{\mathbb{D}}$, and the statement is proved.

$2^{0}$. Proof of limit relation (2.2). The idea is to approximate $\Delta J \cdot R_{0}$ by operators of finite rank and take into account the continuity of perturbation determinants.

Let

$$
\hat{J}_{m}:=\left(\begin{array}{ccc}
J_{m} & \vdots & \\
\cdots & \ldots & \ldots \\
& \vdots & J_{0}
\end{array}\right), \quad \hat{J}_{0, m}:=\left(\begin{array}{ccc}
J_{0, m} & \vdots & \\
\ldots & \ldots & \ldots \\
& \vdots & J_{0}
\end{array}\right)
$$

be the block diagonal operators,

$$
\Delta \hat{J}_{m}=\hat{J}_{m}-\hat{J}_{0, m}=\left(\begin{array}{ccc}
J_{m}-J_{0, m} & \vdots & \\
\cdots & \cdots & \ldots \\
& \vdots & \mathbb{O}
\end{array}\right) .
$$

Since

$$
\Delta J=J-J_{0}=\left(\begin{array}{ccc}
J_{m}-J_{0, m} & \vdots & C \\
\cdots & \cdots & \cdots \\
A & \vdots & J^{(m)}-J_{0}
\end{array}\right),
$$

where the matrices $A$ and $C$ have a single nonzero entry $C_{m 1}=c_{m}-1 / 2, A_{1 m}=$ $a_{m}-1 / 2, A_{j i}=C_{i j}=0, i \neq m, j \neq 1$, then

$$
\left\|\Delta J-\Delta \hat{J}_{m}\right\|_{1} \leq \sum_{j=m}^{\infty}\left\{\left|a_{j}-\frac{1}{2}\right|+\left|b_{j}\right|+\left|c_{j}-\frac{1}{2}\right|\right\} \rightarrow 0, \quad m \rightarrow \infty .
$$

Next, it can be easily seen that $J_{0}-\hat{J}_{0, m}$ has only two nonzero entries. If $h \in \ell^{2}$ then

$$
\left(J_{0}-\hat{J}_{0, m}\right) h=\left(h, e_{m}\right) e_{m+1}+\left(h, e_{m+1}\right) e_{m} \rightarrow 0, \quad m \rightarrow \infty,
$$

or, in other words, $\hat{J}_{0, m} \rightarrow J_{0}$ as $m \rightarrow \infty$ in the strong topology (on each vector form $\left.\ell^{2}\right)$. Let $K$ be a compact subset of $\mathbb{D}$. We can match resolvents of $J_{0}$ and $\hat{J}_{0, m}$ for $z \in K$ :

$$
\left(\hat{J}_{0, m}-\lambda\right)^{-1}-\left(J_{0}-\lambda\right)^{-1}=\left(\hat{J}_{0, m}-\lambda\right)^{-1}\left(J_{0}-\hat{J}_{0, m}\right)\left(J_{0}-\lambda\right)^{-1} .
$$

As $\left\|\left(\hat{J}_{0, m}-\lambda\right)^{-1}\right\|=\left(\operatorname{dist}\left(\lambda(z), \sigma\left(\hat{J}_{0, m}\right)\right)\right)^{-1} \sigma\left(\hat{J}_{0, m}\right)=[-1,1]$, then

$$
\left\|\left(\hat{J}_{0, m}-\lambda\right)^{-1}\right\| \leq C(K), \quad z \in K
$$


Hence $\left(\hat{J}_{0, m}-\lambda\right)^{-1} \rightarrow\left(J_{0}-\lambda\right)^{-1}$ in the strong topology.

According to [5, theorem III.6.3] the convergence of a sequence of operators $A_{m}$ to $A$ in $\mathcal{S}_{1}$ norm and $X_{m}$ to $X$ in the strong topology implies the convergence $A_{m} X_{m}$ to $A X$ in $\mathcal{S}_{1}$. Thereby

$\left(\hat{J}_{m}-\hat{J}_{0, m}\right)\left(\hat{J}_{0, m}-\lambda\right)^{-1} \rightarrow \Delta J R_{0}, \quad I+\left(\hat{J}_{m}-\hat{J}_{0, m}\right)\left(\hat{J}_{0, m}-\lambda\right)^{-1} \rightarrow I+\Delta J R_{0}$

in $\mathcal{S}_{1}$ for each $z$, and hence uniformly on $K$. But the operator $\left(\hat{J}_{m}-\hat{J}_{0, m}\right)\left(\hat{J}_{0, m}-\lambda\right)^{-1}$ has finite rank and

$$
\operatorname{det}\left(I+\left(\hat{J}_{m}-\hat{J}_{0, m}\right)\left(\hat{J}_{0, m}-\lambda\right)^{-1}\right)=\operatorname{det}\left(\frac{J_{m}-\lambda}{J_{0, m}-\lambda}\right)
$$

so the desired result is a consequence of the continuity of perturbation determinants.

\section{REFERENCES}

1. Pavlov B.S. On nonselfadjoint Schrödinger operator I // In "Problems of mathematical physics". LGU, 1966. V. 1. P.102-132 .

2. Pavlov B.S. On nonselfadjoint Schrödinger operator II // In "Problems of mathematical physics". LGU, 1967. V. 2. P.135-157.

3. Bairamov E., Cakar O., Krall A.M. Non-selfadjoint difference operators and Jacobi matrices with spectral singularities// Math. Nachr. 2001. V.229. P.5-14.

4. Geronimo J.S., Case K.M. Scattering theory and polynomials on the real line// Trans. Amer. Math. Soc. 1980. V.258. P.467-494.

5. Gohberg I., Krein M. Introduction to the theory of linear nonselfadjoint operators in Hilbert space // . "Nauka". 1965.

6. Killip R., Simon B. Sum rules for Jacobi matrices and their applications to spectral theory// Ann. Math. 2003. V.158. P.253-321.

7. Carleson L. Sets of uniqueness for functions analytic in the unit disc// Acta Math. 1952. V.87. P.325-345.

8. Aptekarev A.I., Kalyaguine V., Van Assche W. Criterion for the resolvent set of nonsymmetric tridiagonal operators// Proc. Amer. Math. Soc. 1995. V.123. P.2423-2430.

9. Beckermann B. On the convergence of bounded J-fractions on the resolvent set of the corresponding second order difference operator // J. Approx. Theory. 1999. V.99. P.369-408.

10. Beckermann B., Kaliaguine $V$. The diagonal of the Padé table and the approximation of the Weyl function of the second order difference operators // Constr. Approx. 1997. V.13. P.481-510.

11. Beckermann B. Complex Jacobi matrices // J. Comp. Appl. Math. 2001. V.127. P.17-65.

12. Barrios D., Lopez G., Martinez-Finkelstein A., Torrano E. Finite rank approximations of resolvent of infinite banded matrix and continued fractions // Mat. Sbornik. 1999. V.190. No. 4. P. 23-42.

13. Guseinov G. Determination of infinite Jacobi matrix from the scattering data// DAN SSSR. 1976. V.227. No.6. P.1289 -1292.

14. Teschl G. Jacobi Operators and Completely Integrable Nonlinear Lattices. Math. Surveys and Monographs. No.72. 1999. 
15. Fatou P. Séries trigonométriques et séries de Taylor // Acta Math. 1906. V.30. P.335-400.

16. Beurling A. Ensembles exceptionneles// Acta Math. 1939. V.72. P.1-13.

17. Khrushchev $S$. The problem of simultaneous approximation and erasure of singularities for Cauchy type integrals// Trudy MIAN SSSR. 1978. V.130. P.124195.

18. Besicovitch A.S., Taylor S.J. On the complementary intervals of a linear closed sets of zero Lebesque measure // J. Lond. Math. Soc. 1954. V.29. P.449-459.

19. Taylor B.A., Williams D.L. Boundary zero sets of $A^{\infty}$ functions satisfying growth conditions // Proc. Amer. Math. Soc. 1972. V.35. P.155-160.

20. Korenblum B. Quasi-analytic classes of functions in a disk // DAN SSSR 1965. V.164. P.36-39.

21. Nikishin. The discrete Sturm-Liouville operatr and some probof the function theory // Trudy Petrovsky seminar. 1984. V. 10. P.3-77.

22. Toda . Theory of nonlinear lattices. Moscow: "Mir". 1984.

23. Kaliagin $V$. On rational approximation of the resolvent function of difference second order operator // UMN. 1994. V.49. No. 3. P.181-182.

24. Yosida K. Functional analysis// Springer-Verlag. 1965.

25. Geronimo J.S. A relation between the coefficients in the recurrence formula and the spectral function for orthogonal polynomials // Trans. Amer. Math. Soc. 1980. V.260. No.1. P.65-82.

26. Kuijlaars A., McLaughlin K.T.-R., Van Assche W., Vanlessen M. The RiemannHilbert approach to strong asymptotics for orthogonal polynomials on $[-1,1] / /$ Adv. in Math. 2004. V. 188. P.337-398.

Institute for Low Temperature Physics and Engineering, 47, Lenin ave., Kharkiv, UKRAINE

E-mail address: egorova@ilt.kharkov.ua

E-mail address: golinskii@ilt.kharkov.ua 